\title{
Identification with Stochastic Sampling Time Jitter
}

Frida Eng, Fredrik Gustafsson

Division of Automatic Control

E-mail: frida@isy.liu.se, fredrik@isy.liu.se

6th December 2006

Report no.: LiTH-ISY-R-2757

Submitted to Automatica

Address:

Department of Electrical Engineering

Linköpings universitet

SE-581 83 Linköping, Sweden

WWW: http://www.control.isy.liu.se

AUTOMATIC CONTROL

REGLERTEKNIK

LINKÖPINGS UNIVERSITET

Technical reports from the Automatic Control group in Linköping are available at http://www. control.isy.liu.se/publications. 


\begin{abstract}
This work investigates how stochastic unmeasurable sampling jitter noise affects the result of system identification, and proposes a modification of known approaches to mitigate the effects of sampling jitter. By just assuming conventional additive measurement noise, the analysis shows that the identified model will get a bias in the transfer function amplitude that increases for higher frequencies. A frequency domain approach with a continuous time system model allows an analysis framework for sampling jitter noise. The bias and covariance in the frequency domain model are derived. This is used in bias compensated (weighted) least squares algorithms, and by asymptotic arguments this leads to a maximum likelihood algorithm. Continuous time output error models are used for numerical illustrations.
\end{abstract}

Keywords: system identification, stochastic systems, least-squares estimation, maximum likelihood, frequency domain 


\title{
Identification with Stochastic Sampling Time Jitter *
}

\author{
Frida Eng ${ }^{1}$, Fredrik Gustafsson.
}

Dept of EE, Linköpings universitet, SE-58183 Linköping, Sweden

\begin{abstract}
This work investigates how stochastic unmeasureable sampling jitter noise affects the result of system identification, and proposes a modification of known approaches to mitigate the effects of sampling jitter. By just assuming conventional additive measurement noise, the analysis shows that the identified model will get a bias in the transfer function amplitude that increases for higher frequencies. A frequency domain approach with a continuous time system model allows an analysis framework for sampling jitter noise. The bias and covariance in the frequency domain model are derived. This is used in bias compensated (weighted) least squares algorithms, and by asymptotic arguments this leads to a maximum likelihood algorithm. Continuous time output error models are used for numerical illustrations.
\end{abstract}

Key words: system identification, stochastic systems, least-squares estimation, maximum likelihood, frequency domain

\section{Introduction}

Consider a deterministic signal model $s(t ; \theta)$, which may depend on an observed input. This work studies the problem of identifying the unknown parameter vector $\theta$, when the discrete time observations $y_{k}$ requested at time $t=k T$ ( $T$ denotes the sampling interval) are subject both to the usual additive measurement noise and also stochastic unmeasurable jitter noise $\tau_{k}$. That is, the observation includes the term $s\left(k T+\tau_{k} ; \theta\right)$, which becomes a stochastic variable.

This type of non uniform sampling may occur when uniform sampling is requested, but the sensor for one or several reasons cannot measure exactly at that time instant, and the true sampling time is either unmeasurable, or the communication protocol does not allow to transport time stamps to each measurement. Sampling jitter may also occur due to imperfect hold circuits, synchronization or other hardware problems. Not even highperformance digital oscilloscopes are free from sampling jitter as demonstrated in [15]. They developed a dedicated system identification experiment to estimate jitter

\footnotetext{
* Parts of this work were presented at The IFAC World Congress, 2005, in Prague, and at The IFAC Symposium on System Identification, 2006, in Newcastle.

Email addresses: frida@isy.liu.se (Frida Eng), fredrik@isy.liu.se (Fredrik Gustafsson).

1 Corresponding author Mrs. Eng previously published as Miss Gunnarsson. Tel. +4613 281365. Fax +4613282622.
}

effects, and the result is that a commercial sampling oscilloscope with 20 ps sampling time has a sampling jitter standard deviation of around $1 \mathrm{ps}$, that is $5 \%$ of the sampling time.

The general problem of nonuniform sampling is extensively treated in literature, see [1] and [10]. In most publications, the sampling times are known, and the problem is to analyze leakage and alias effects. Another twist is to design sampling times to minimize aliasing. For stochastic sampling jitter, the distribution of $s\left(t+\tau_{k}\right)$ is derived in $[2,3]$ and [14]. These results will be used and extended in this paper.

The basic idea is as follows. The frequency domain approach is to minimize the distance between the measurement and signal model Discrete Time Fourier Transforms (DTFT), $\| \operatorname{DTFT}\left(y_{k}\right)-\operatorname{DTFT}\left(s\left(t+\tau_{k} ; \theta\right) \|\right.$ with respect to the parameters $\theta$ in some suitable norm. This frequency domain approach is standard in system identification $[11,9]$. A continuous time model for $s(t)$ is used to be able to analyze the sampling jitter effects. The analysis shows that by neglecting the jitter, the Fourier Transform (FT) of the signal model will suffer from an amplitude bias in $|\mathrm{FT}(s(t))|=|S(f)|$ that increases for higher frequencies. Further, the larger jitter noise variance, the larger bias. The remedy is to compensate for the bias, and the closed form solution involves a frequency weighting window in the norm, $\| \operatorname{DTFT}\left(y_{k}\right)-$ $\int S(\psi) w(f-\psi) d \psi \|$.

The outline is as follows. The system identification prob- 
lem and main notation are presented in Section 2. In Section 3 , the bias effect of sampling jitter noise on the frequency transform is derived, and the bias compensated least squares estimator is proposed. Section 4 derives the second order properties of the frequency transform due to jitter noise, and a weighted least squares algorithm as well as an asymptotic maximum likelihood estimator are presented. Section 5 illustrates these algorithms for several simulated numerical examples. The work is concluded in Section 6.

\section{Problem Formulation}

The general problem formulation looks as follows. The sensor is requested to sample uniformly, but delivers discrete time measurements corrupted by amplitude noise and sampling time jitter according to

$$
y_{k}=s\left(k T+\tau_{k} ; \theta\right)+v\left(k T+\tau_{k} ; \theta\right) .
$$

The signal term, noise term and jitter distribution can all be dependent on the unknown parameter $\theta$ and given by

$$
\begin{gathered}
s(t ; \theta)=g_{\theta} * u(t), \\
v(t ; \theta)=h_{\theta} * e(t), \\
\tau_{k} \in p_{\theta}(\tau) .
\end{gathered}
$$

Here $u$ is a known input, $e$ is white noise with known characteristics, $g_{\theta}(t)$ denotes the system impulse response and $h_{\theta}(t)$ the noise dynamics. The jitter sampling noise is a sequence of independent stochastic variables with probability density function (pdf) $p_{\theta}(\tau)$. Both the signal, noise and sampling models can be parameterized in the unknown parameter vector $\theta$. We will primarily focus on continuous time systems here.

The system identification problems under consideration can be stated as estimating the parameter $\theta$ in a model structure

$$
\begin{aligned}
& \mathcal{M}_{O E}: g_{\theta}(t), h_{\theta}(t)=\delta(t), p_{\theta}(\tau)=p(\tau), \\
& \mathcal{M}_{B J}: g_{\theta}(t), h_{\theta}(t), p_{\theta}(\tau)=p(\tau), \\
& \mathcal{M}_{J O E}: g_{\theta}(t), h_{\theta}(t)=\delta(t), p_{\theta}(\tau), \\
& \mathcal{M}_{J B J}: g_{\theta}(t), h_{\theta}(t), p_{\theta}(\tau) .
\end{aligned}
$$

Here, OE denotes the output error and BJ the BoxJenkins model structure, respectively, where the jitter distribution is known. JOE and JBJ are the corresponding problems where also the jitter noise distribution is parametrized.

Using previous knowledge about the sampling jitter effect in the frequency domain indicates that the frequency domain approach, e.g., [11,9], is suitable for identification in this case. Denote the Fourier transform of the measurements and signal model, respectively,

$$
\begin{aligned}
Y_{d}(f) & =\sum y_{k} e^{-i 2 \pi f k T}, \\
S(f ; \theta) & =G(f ; \theta) U(f) .
\end{aligned}
$$

The general problem formulation is now to minimize the distance between the measurement, $Y_{d}(f)$, and model, $S(f ; \theta)$, in the frequency domain.

$$
\hat{\theta}=\arg \min _{\theta} \int \lambda(f ; \theta)\left|Y_{d}(f)-S(f ; \theta)\right|^{2} \mathrm{~d} f,
$$

for some suitable weighting function. Normally, [11,9], the weights, $\lambda(f ; \theta)$ are given by the inverse noise spectrum. We will show a few other examples later.

Given a continuous signal model $S_{c}(f)$, a well known property of the Fourier transform gives that the discrete Fourier Transform, $S_{d}(f)$, becomes

$$
S_{d}(f)=\int S_{c}(\psi) d_{N}(f-\psi) \mathrm{d} \psi
$$

Here $d_{N}(f)$ is the normalized Dirichlet kernel (also called the aliased sinc function), defined as

$$
d_{N}(f)=e^{-i \pi f(N-1) T} \frac{\sin (\pi f N T)}{\sin (\pi f T)}
$$

The local behavior of the normalized Dirichlet kernel (see Fig. 1 on p. 4) describes the effects of leakage and its $1 / T$ periodicity describes aliasing. For the regular case, with no sampling jitter, the correct way is to compare $Y_{d}(f)$ with $S_{d}(f ; \theta)$, and using the unweighted least squares (LS) norm in (5) over a discrete set of frequencies yields the parameter vector $\theta$ as

$$
\hat{\theta}^{L S}=\arg \min _{\theta} \sum_{f}\left|Y_{d}(f)-\int S_{c}(\psi ; \theta) d_{N}(f-\psi) \mathrm{d} \psi\right|^{2} .
$$

Similarly to [12] and [5], the frequency set under consideration is arbitrary, in order to have the freedom to highlight certain frequency regions of interest.

This problem is closely related to the approach $[7,6]$ for the special case of no jitter noise, and [5] for the case of non-uniform sampling (but deterministic known sampling times). Note also that the leakage can also be interpreted as originating from a transient in the signal model caused by an unknown initial state. An alternative dual approach is based on estimating this initial state is studied in $[13,11]$.

The main idea with bias compensation is to mitigate the bias effects in $Y_{d}(f)$ by replacing the deterministic discrete time signal model $\int S_{c}(\psi ; \theta) d_{N}(f-\psi) d \psi$ in (8) 
with a term, that besides sampling effects also includes the jitter noise. This term is denoted $\mu_{Y}(f)$, and is derived from $E\left(Y_{d}(f)\right)$.

The purpose of this work is to analyze the bias and variance contribution from jitter sampling noise on the signal model $S_{c}(f ; \theta)$, and modify the criterion (8) accordingly.

\section{Bias Compensation}

First, the bias in $Y_{d}(f)$ due to sampling jitter is derived. Then, the bias compensated LS (BCLS) estimate is defined. Equation (8) is here replaced by the more general expression

$$
\hat{\theta}=\arg \min _{\theta} \sum_{f}\left|Y_{d}(f)-\mu_{Y}(f ; \theta)\right|^{2}
$$

where $\mu_{Y}(f ; \theta)$ is to be derived. In the following two subsections, the dependence on the parameter vector $\theta$ will be implicit.

\subsection{Bias in $Y_{d}(f)$}

The DTFT (3) of the sequence $y_{k}$ using the FT $S(f)=$ $\int s(t) e^{-i 2 \pi f t} d t$ and the sampling jitter model (1) can be written

$$
\begin{aligned}
Y_{d}(f) & =\sum_{k=0}^{N-1} y_{k} e^{-i 2 \pi f k T} \\
& =\sum_{k=0}^{N-1}\left(s\left(k T+\tau_{k}\right)+v_{k}\right) e^{-i 2 \pi f k T} \\
& =\hat{S}_{d}(f)+\underbrace{\sum_{k=0}^{N-1} v_{k} e^{-i 2 \pi f k T}}_{\hat{V}(f)} .
\end{aligned}
$$

Using the inverse FT $s(t)=\int S_{c}(f) e^{i 2 \pi f t} d t$, the signal term becomes

$$
\begin{aligned}
\hat{S}_{d}(f) & =\sum_{k=0}^{N} s\left(k T+\tau_{k}\right) e^{-i 2 \pi f k T} \\
& =\sum_{k=0}^{N-1} \int S_{c}(\psi) e^{i 2 \pi \psi\left(k T+\tau_{k}\right)} \mathrm{d} \psi e^{-i 2 \pi f k T} \\
& =\int S_{c}(\psi) W(f, \psi) \mathrm{d} \psi,
\end{aligned}
$$

where

$$
W(f, \psi) \triangleq \sum_{k=0}^{N-1} e^{i 2 \pi(\psi-f) k T} e^{i 2 \pi \psi \tau_{k}}
$$

The term $W$ is a stochastic frequency window, completely describing the jitter, leakage and aliasing effects in the signal term $S_{d}(f)$ of $Y_{d}(f)$. Note that $W(f, \psi)=$ $d_{N}(f-\psi)$ in the special case of no jitter, $\tau_{k}=0$. It should be stressed that this continuous time frequency domain approach is perhaps the only way to explicitly separate the signal amplitude noise, captured in $\hat{V}$, and sampling jitter noise, captured in $W$.

\subsection{First moment of $W$}

The moments of the transform $Y_{d}(f)$ have an explicit dependence on the signal transform $S_{c}(f)$. The mean value of $Y_{d}(f)$ in $(3)$ is

$$
\begin{aligned}
\mu_{Y}(f) & =\mathrm{E}\left[Y_{d}(f)\right]=\mathrm{E}\left[\hat{S}_{d}(f)\right] \\
& =\int S_{c}(\psi) \mathrm{E}[W(f, \psi)] \mathrm{d} \psi \\
& =\int S_{c}(\psi) \mu_{W}(f, \psi) \mathrm{d} \psi
\end{aligned}
$$

and $\mu_{W}=E[W]$ is given by the following lemma.

Lemma 1 (Mean value) The mean value, $\mu_{W}$, with respect to $\tau_{k}$, of the stochastic window, $W(f, \psi)$, defined in Eq. (12), is

$$
\mu_{W}(f, \psi)=\varphi(-\psi) d_{N}(f-\psi),
$$

where $\varphi(f)$ is the characteristic function $\varphi(f)=$ $E\left[e^{-i 2 \pi f \tau}\right]$ of $\tau$ and $d_{N}(f)$ is the normalized Dirichlet kernel $(7)$.

\section{PROOF.}

$$
\begin{aligned}
\mu_{W}(f, \psi) & =\mathrm{E}[W(f, \psi)], \\
& =\sum_{k=0}^{N-1} e^{i 2 \pi(\psi-f) k T} \mathrm{E}\left[e^{i 2 \pi \psi \tau_{k}}\right] \\
& =\varphi(-\psi) \underbrace{\frac{1-e^{i 2 \pi(\psi-f) N T}}{1-e^{i 2 \pi(\psi-f) T}}}_{d_{N}(f-\psi)},
\end{aligned}
$$

where we recognize the characteristic function, $\varphi(f)=$ $E\left[e^{-i 2 \pi f \tau}\right]$ and the normalized Dirichlet kernel, $d_{N}$.

The characteristic function $\varphi(f)=\varphi(f ; \theta)$ models damping corresponding to the sampling jitter noise. It will include possible distribution parameters from $p_{\theta}(\tau)$ in (2). Its properties include:

- $|\varphi(f)| \leq 1, \forall f$,

- $\varphi(f)=1$ if and only if there is no jitter noise, so $\tau_{k}=0, \forall k$ 
- otherwise $|\varphi(f)| \rightarrow 0$, when $f \rightarrow \infty$.

- The decay rate of $|\varphi(f)|$ increases with the jitter noise variance, for a given class of distributions.

The second property implies that $\mu_{W}(f, \psi)=d_{N}(f-\psi)$ for the case of no jitter noise.

\subsection{Bias Compensated Least Squares Estimate}

Since now $\mu_{Y}(f)=\mathrm{E}\left[Y_{d}(f)\right]$ is completely known, we can compensate for both the leakage and jitter effect on the measurement DTFT. We include this in the parameter estimation as, cf. (9),

$$
\hat{\theta}^{B C L S}=\arg \min _{\theta} \sum_{f}\left|Y_{d}(f)-\mu_{Y}(f ; \theta)\right|^{2}
$$

where

$$
\mu_{Y}(f ; \theta)=\int S_{c}(\psi ; \theta) \varphi(-\psi ; \theta) d_{N}(f-\psi) \mathrm{d} \psi .
$$

Both the signal $S_{c}(f ; \theta)$ and jitter pdf $p(\tau ; \theta)$, and thus characteristic function $\varphi(f ; \theta)$, may depend on the unknown parameter vector $\theta$.

\subsection{Illustrations}

Let us consider the implications of Lemma 1 in more detail. The Dirichlet kernel that appears as the window function in the jitter-free case is illustrated in Fig. 1 (solid black). As two concrete examples of characteristic functions, consider

$$
\begin{array}{lll}
\tau \in U(-1,1) & \Rightarrow & \varphi(f)=\frac{\sin (2 \pi f)}{2 \pi f} \\
\tau \in N(0,1 / 3) & \Rightarrow & \varphi(f)=e^{-2 \pi^{2} f^{2} / 3},
\end{array}
$$

which have the same variance. The amplitudes of these functions are also illustrated in Fig. 1.

According to Lemma 1, the jitter noise will attenuate the amplitude of $S_{d}(f)$. The attenuation is inversely linear in frequency for uniform jitter and exponentially decaying for Gaussian jitter noise. This also implies that frequencies above the Nyqvist frequency are attenuated, surpressing alias. This attenuation is compensated for in the BCLS estimate.

Consider now a single frequency signal with known amplitude and phase, but unknown frequency, $f_{0}, s(t)=$ $\sin \left(2 \pi f_{0} t\right)$, such that $S_{c}(f)=1 / 2 i\left(\delta\left(f-f_{0}\right)-\delta\left(f+f_{0}\right)\right)$. We compare uniform sampling, $T=2$, and jitter sampling, $\tau \in U(-T / 2, T / 2)$, for two different frequencies

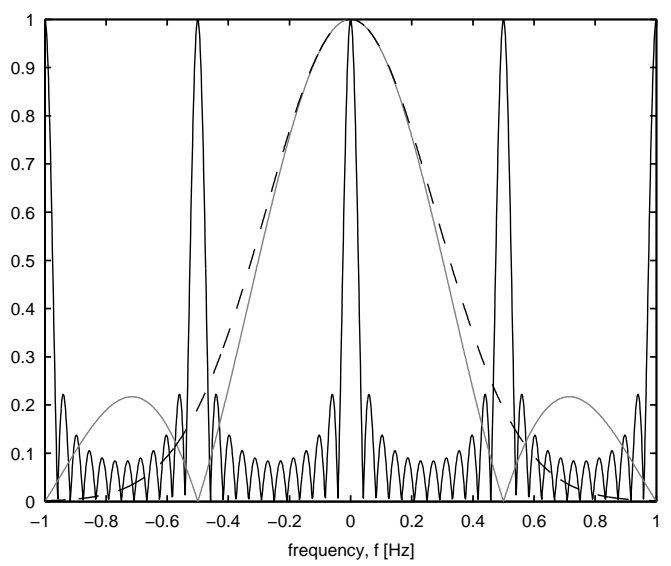

Fig. 1. Example of the normalized Dirichlet kernel, $d_{N}(0.25-f)$ (solid black), (7), and the two characteristic functions in (15), (a) solid gray and (b) dashed.

$f_{0}$, and get the following expressions on the positive frequency axis. Uniform sampling gives

$$
\begin{aligned}
Y_{d}(f) & =\frac{1}{2 i} d_{N}(f-0.14), & f_{0} & =0.14 \\
Y_{d}(f) & =\frac{1}{2 i} d_{N}(f-1.14), & f_{0} & =1.14,
\end{aligned}
$$

where $d_{N}(f-0.14)=d_{N}(f-1.14)$. When the sampling times are corrupted by jitter noise, (13) and Lemma 1 give

$$
\begin{aligned}
& \mathrm{E}\left[Y_{d}(f)\right]=\frac{1}{2 i} \varphi(-0.14) d_{N}(f-0.14), \quad f_{0}=0.14 \\
& \mathrm{E}\left[Y_{d}(f)\right]=\frac{1}{2 i} \varphi(-1.14) d_{N}(f-1.14), \quad f_{0}=1.14 .
\end{aligned}
$$

The result is still periodic, but with different damping depending on the frequency.

This is also shown in Fig. 2, where $\left|Y_{d}(f)\right|$ and $\left|\mathrm{E}\left[Y_{d}(f)\right]\right|$ are plotted for the different cases in (16). From Fig. 1, we know that the amplitude bias is larger for higher frequencies $f_{0}, 0<\varphi(-1.14)<\varphi(-0.14)<1$, which is why we can separate the two frequencies in this jitter sampling case.

\section{Covariance Compensation}

Bias compensating $Y(f)$ implies unbiased estimates of $S_{c}(f ; \theta)$. To reach asymptotic efficiency, also the variance of $Y(f)$ is needed. In this section, both a Weighted Least Squares (WLS) and Maximum Likelihood (ML) estimates are stated based on this covariance. 


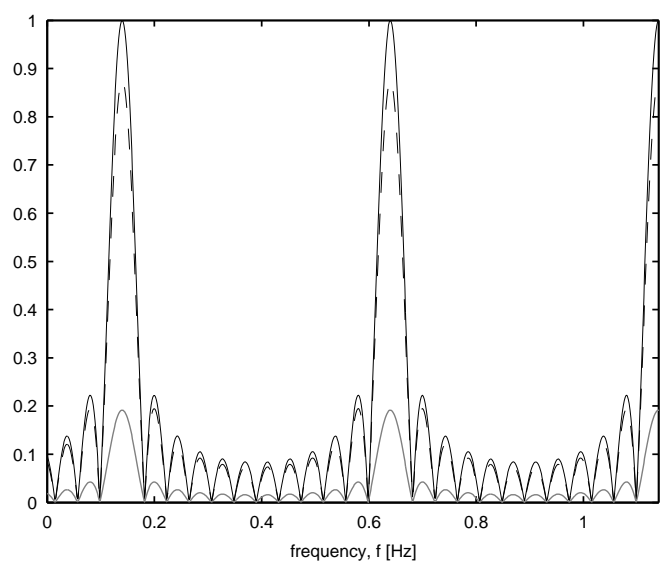

Fig. 2. $\left|\mathrm{E}\left[Y_{d}(f)\right]\right|$ given in (16) are shown, (a,b) thin line overlap, while (c) dashed and (d) thick gray are different.

\subsection{Covariance of $Y_{d}(f)$}

To use these two estimators we need the covariance of $Y, R_{Y}$, which we get from the expressions in (10) as

$$
R_{Y}(f, \psi)=R_{\hat{S}}(f, \psi)+R_{\hat{V}}(f, \psi)
$$

with

$$
\begin{aligned}
& R_{\hat{S}}(f, \psi)=\operatorname{Cov}(\hat{S}(f), \hat{S}(\psi)) \\
& =\iint S(\eta) \operatorname{Cov}(W(f, \eta), W(\psi, \zeta)) S(\zeta)^{*} \mathrm{~d} \eta \mathrm{d} \zeta \\
& =\iint S(\eta) R_{W}(f, \eta, \psi, \zeta) S(\zeta)^{*} \mathrm{~d} \eta \mathrm{d} \zeta .
\end{aligned}
$$

Here, $R_{W}(f, \eta, \psi, \zeta) \triangleq \operatorname{Cov}(W(f, \eta), W(\psi, \zeta))$ is the covariance of the stochastic frequency window in Eq. (12). The contribution from the measurement noise is

$$
\begin{aligned}
R_{\hat{V}}(f, \psi) & =\operatorname{Cov}(\hat{V}(f), \hat{V}(\psi))), \\
& =\sigma^{2} \sum_{k=0}^{N-1} e^{-i 2 \pi(f-\psi) k T},
\end{aligned}
$$

since we restricted the investigation to white Gaussian measurement noise.

\subsection{Covariance of $W(f)$}

The covariance of $W(f)$ is

$R_{W}(f, \eta, \psi, \zeta)=\mathrm{E}\left[W(f, \eta) W^{*}(\psi, \zeta)\right]-\mu_{W}(f, \eta) \mu_{W}(\psi, \zeta)^{*}$

and the second order moment is given by the following lemma.
Lemma 2 (Second order moment) The second order moment of $W$ is given as

$$
\mathrm{E}\left[W(f, \eta) W^{*}(\psi, \zeta)\right]=\Phi^{T}(\eta, \zeta) D_{N}(f-\eta, \psi-\zeta) .
$$

where

$$
\Phi(f, \psi)=\left(\begin{array}{c}
\varphi(-f) \varphi(\psi) \\
\varphi(\psi-f) \\
\varphi(-f) \varphi(\psi)
\end{array}\right)
$$

and

$$
D_{N}(f, \psi)=\left(\begin{array}{c}
\sum_{k=0}^{N-1} \sum_{l=0}^{k-1} e^{-i 2 \pi(f k-\psi l) T} \\
d_{N}(f-\psi) \\
\sum_{k=0}^{N-1} \sum_{l=0}^{k-1} e^{-i 2 \pi(-\psi k+f l) T}
\end{array}\right)
$$

The functions were defined in Lemma 1

PROOF. The second moment of the stochastic window, $W$, is by definition

$$
\begin{aligned}
E & {\left[W(f, \eta) W^{*}(\psi, \zeta)\right] } \\
& =\sum_{k, l} e^{i 2 \pi(\eta-f) k T} \mathrm{E}\left[e^{i 2 \pi\left(\eta \tau_{k}-\zeta \tau_{l}\right)}\right] e^{-i 2 \pi(\zeta-\psi) l T} \\
& =\sum_{k} \sum_{l<k}+\sum_{k} \sum_{l=k}+\sum_{k} \sum_{l>k} \\
& =V_{1}+V_{2}+V_{3} .
\end{aligned}
$$

The first term of the second moment above is

$$
\begin{aligned}
& V_{1}(f, \psi, \eta, \zeta) \\
& \quad=\varphi(-\eta) \varphi(\zeta) \sum_{k=0}^{N-1} e^{i 2 \pi(\eta-f) k T} \sum_{l=0}^{k-1} e^{-i 2 \pi(\zeta-\psi) l T}
\end{aligned}
$$

since $\varphi(f)=\mathrm{E}\left[e^{-i 2 \pi f \tau_{k}}\right]$ was the $\mathrm{CF}$ of $\tau_{k}$ and $\tau_{k}$ are i.i.d. The second term is the sum over $l=k$,

$$
\begin{aligned}
V_{2}(f, \psi, \eta, \zeta) & =\sum_{k=0}^{N-1} e^{i 2 \pi(\eta-f-\zeta+\psi) k T} \varphi(\zeta-\eta) \\
& =\varphi(\zeta-\eta) d_{N}(f-\eta-\psi+\zeta)
\end{aligned}
$$

and, finally, the third term is

$$
\begin{aligned}
& V_{3}(f, \psi, \eta, \zeta) \\
& \quad=\varphi(-\eta) \varphi(\zeta) \sum_{l=0}^{N-1} e^{-i 2 \pi(\zeta-\psi) l T} \sum_{k=0}^{l-1} e^{i 2 \pi(\eta-f) k T}
\end{aligned}
$$


Identification of terms gives the result of the Lemma.

The two factors, $\Phi$ and $D_{N}$, correspond to parts depending on the sampling noise and on the finite sampling, respectively, cf. $\varphi(f)$ and $d_{N}(f)$ in Lemma 1. It is also quite straightforward to verify $R_{W}(f, \eta, \psi, \zeta)=0$ when there is no sampling jitter present, since in that case, $E\left[W(f, \eta) W^{*}(\psi, \zeta)\right]=d_{N}(f-\eta) d_{N}(\psi-\zeta)^{*}=$ $\mu_{W}(f, \eta) \mu_{W}(\psi, \zeta)^{*}$, and (19) evaluates to zero.

\subsection{Weighted Least Squares}

The jitter noise will perturb higher frequencies more than lower frequencies, which can be mitigated with the Weighted Least Squares (WLS) approach, see e.g., [8, Ch. 2], where the weights, $\lambda(f)$, are given by the covariance. Furthermore, the correlation between different frequencies needs to be taken into account. That is, (9) is here extended to

$$
\begin{aligned}
& \hat{\theta}^{W B C L S} \\
& \quad=\arg \min _{\theta}\left\{\left(Y-\mu_{Y}(\theta)\right)^{*} R_{Y}(\theta)^{-1}\left(Y-\mu_{Y}(\theta)\right)\right\},
\end{aligned}
$$
where $R_{Y}=I$ corresponds to $(9)$. Here $Y=\left(Y_{d}\left(f_{1}\right), \ldots, Y_{d}\left(f_{n}\right)\left(I_{J, O E}\right.\right.$ example, both with second order systems, are $\mu_{Y}=E[Y]$ from (13) and Lemma 1 , and $R_{Y}=\operatorname{Cov}(Y)$ as given in (17), (19) and Lemma 2.

\subsection{Maximum Likelihood}

The central limit theorem together with the fact that the sampling noise $\tau_{k}$ and the measurement noise $v_{k}$ are i.i.d., Equations (10) and (11) indicate that both $\hat{V}$ and $\hat{Y}_{d}$ are asymptotically Gaussian, and hence

$$
Y_{d} \in A s \mathcal{N}\left(\mu_{Y}, R_{Y}\right)
$$

where $A s \mathcal{N}$ denotes the asymptotic Gaussian distribution. When this holds, the asymptotic Maximum Likelihood (ML) estimator, see e.g., [9, Ch. 7] or [4], can be defined as

$$
\begin{aligned}
& \hat{\theta}^{M L} \\
& \begin{aligned}
=\arg \min _{\theta}\{ & \left(Y-\mu_{Y}(\theta)\right)^{*} R_{Y}(\theta)^{-1}\left(Y-\mu_{Y}(\theta)\right)+ \\
& \left.+\ln \operatorname{det} R_{Y}(\theta)\right\},
\end{aligned}
\end{aligned}
$$

with the vector $Y$, the mean $\mu_{Y}$ and variance $R_{Y}$ given in the previous section.

\subsection{Implementation issues}

In the examples in Sec. 5, a grid based search is implemented to avoid problems with local minima. However,

a promising alternative would be to use numerical methods to minimize the estimators in (20) and (22), like gradient or Gauss-Newton searches as described in [9]. To avoid potential problems with local minima, the parameter vector can be initialized by standard methods for OE models [9] neglecting the jitter noise. This should work fine for moderate levels of jitter noise.

One way to avoid the matrix inversion of $R_{Y}$ is to only consider frequencies $f=n / N T$ for integer valued $n$. The idea is that the Fourier transform $Y_{d}(f)$ is asymptotically uncorrelated [9] for these frequencies, which would simplify the estimators considerably. For instance, the ML estimator becomes

$$
\hat{\theta}^{M L}=\arg \min _{\theta} \sum_{n}\left\{\frac{\left|Y_{d}(n / N T)-\mu_{Y}(n / N T ; \theta)\right|^{2}}{R_{Y}(n / N T ; \theta)}+\ln \operatorname{det} R_{Y}(n / \Lambda\right.
$$

\section{Examples}

Three numerical examples are presented. First, a known first order OE model is used to illustrate that the jitter noise distribution is identifiable. Then, a $\mathcal{M}_{O E}$ and a presented to illustrate the main results.

To find the best parameter vector $\theta$ the maximum likelihood estimator (22) will be used. The DTFT is calculated for the frequencies $f_{k}=k / N T, k=0, . .\lfloor N / 2\rfloor-1$, i.e., we get the DFT. In the examples we will also use the least squares estimator (14) to compare with.

The input $u(t)$ is a Pseudo Random Binary Sequence (PRBS) signal, which jumps between 0 and 1 at time intervals of exponentially distributed lengths, with a mean value of $8 \mathrm{~s}$. The measurement noise $v(t)$ is zero-mean Gaussian with standard deviation 0.01 .

\subsection{Known first order OE model with unknown jitter $p d f$}

For this example we chose

$$
\mathcal{M}: g_{\theta}(t)=\mathcal{L}^{-1}\left(\frac{1}{s+2}\right), h_{\theta}(t)=\delta(t), p_{\theta}(\tau)
$$

where only $p_{\theta}(t)$ is parameter dependent. We use a known first order system, where the nput and measurement noise were defined above. In Fig. 3, an example of the input $u(t)$ and output $y(t)$ is given for the above settings.

The focus is on finding the distribution of the sampling noise. We choose a parameterization based on a 


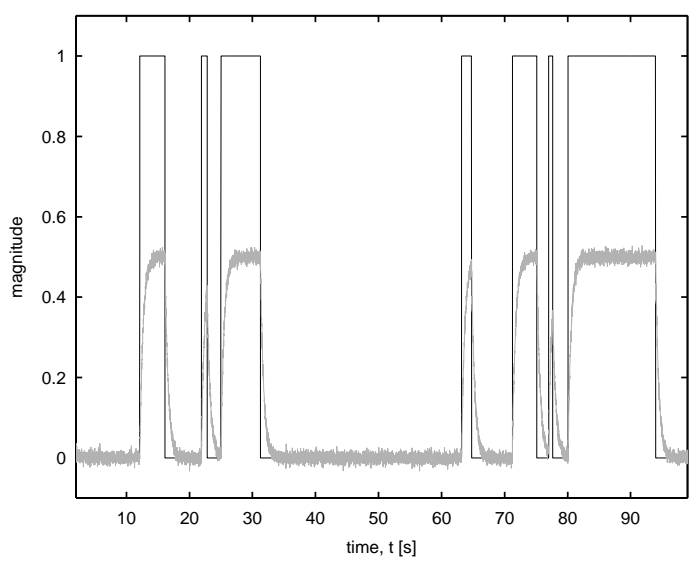

Fig. 3. Example of a PRBS signal $(u(t)$ black $)$ and the output from $G(s)=1 /(s+2),(y(t)$ gray $)$, in continuous time.

sum of $n$ uniformly distributed variables in the interval $[-a / n, a / n]$. That is, the support of the pdf $p_{\theta}(\tau)$ is always $[-a, a]$, and its parameters are $\theta=[n, a]$. The characteristic function of $p_{\theta}(\tau)$ can be written as

$$
\varphi_{\theta}(f)=\left(\frac{\sin \left(\frac{\pi f a}{n+1}\right)}{\frac{\pi f a}{n+1}}\right)^{n+1}=\operatorname{sinc}\left(\frac{f a}{n+1}\right)^{n+1},
$$

since a sum of independent stochastic variables corresponds to convolving their pdf's, which in turns corresponds to multiplying their characteristic functions. The expected value is 0 and the variance is $\frac{1}{n+1} \frac{a^{2}}{3}$. Setting $n=0$ yields a rectangular distribution, $n=1$ a triangular distribution, and increasing $n$ makes the distribution converging to a normal distribution. Thus, a higher $n$ indicates a more narrow distribution. See Fig. 4 for a few examples of the pdf and corresponding (of equal variance) Gaussian distribution.

Now, we want to test both jitter covering the whole interval $[-T / 2, T / 2]$, as well as only a portion of it; change the order but keep the variance fixed; and also look outside the model class. Therefore, we use $p_{\theta}(\tau)$ given by the $\mathrm{CF}$ in Eq. (25), for four sets of true parameters $\left[n^{0}, a^{0}\right]=[0, T / 2],[0, T / 2 \sqrt{3}],[2, T / 2]$, and $[2, T / 2 \sqrt{3}]$, with sampling time $T=1$. This gives the variance of the jitter as $\left(\sigma^{0}\right)^{2}=(T / 2 \sqrt{3})^{2},(T / 6)^{2},(T / 6)^{2}$ and $(T / 6 \sqrt{3})^{2}$, respectively. We also let the true pdf be a zero-mean Gaussian distribution with the same variance as the two last cases above, namely $\sigma^{2}=(T / 6)^{2}$ and $\sigma^{2}=(T / 6 \sqrt{3})^{2}$.

For the six different pdf's, we evaluate the mean value and the standard deviation of the estimated parameters, $\hat{\theta}$, based on 128 different realizations of the sampling noise sequence and the input. The result is shown in Table 1 , for both the ML and LS estimate. We also compute the estimate of the standard deviation $\sigma$, implied by $\hat{n}$ and $\hat{a}$. We can conclude the following:

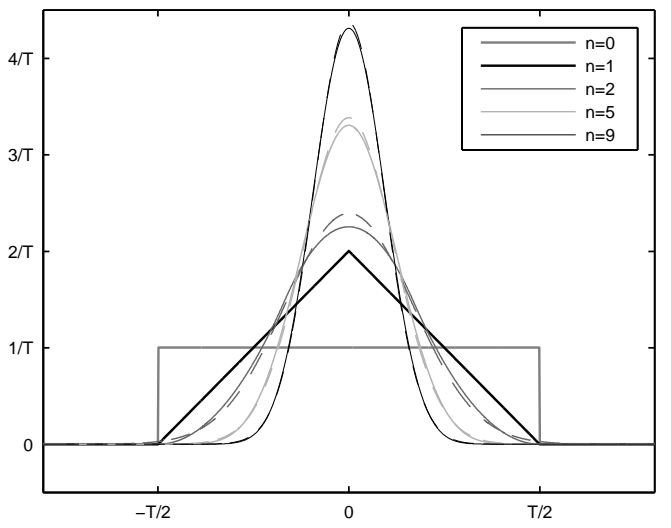

Fig. 4. The solid lines show the pdf, $p_{\theta}(\tau)$, corresponding to different values of $\theta=[n, T / 2]$, when the $\mathrm{CF}$ is $\varphi(f)=\operatorname{sinc}\left(\frac{f a}{n+1}\right)^{n+1}$. For the higher values of $n$, the dashed lines show the Gaussian bells with the same variance as the parameterized pdf. The difference is almost invisible in this plot for $n=9$.

- The standard deviation $\sigma$ for the sampling noise as accurately estimated, while the exact parameter constellation, $[n a]$, is harder. This can be due to too few samples $(N=200)$ to be able to estimate the shape of the distribution.

- The distribution with $n^{0}=0, a^{0}=0.5$, rectangular over the whole interval, has best results.

- The ML estimate has the smallest error for all cases, indicating that the covariance $R_{Y}$ vary significantly along the frequency axis..

- True pdf's outside the model class (last two tests) pose no problem to the estimation of $\sigma$.

\subsection{Unknown second order OE model with known jitter $p d f$}

Now, let us focus on the linear system, $g(t)$, that is, the $\mathcal{M}_{O E}$ problem in (2a). We illustrate the algorithms for a second order system, specified by its transfer function $G$. The model is

$$
\mathcal{M}: g_{\theta}(t), h_{\theta}(t)=\delta(t), p_{\theta}(\tau)=p(\tau)
$$

The system $g_{\theta}(t)=\mathcal{L}^{-1}\left(G_{\theta}(s)\right)$ is parameterized as

$$
\begin{aligned}
G_{\theta}(s) & =k_{0} \frac{s+z}{\left(s-\left(p_{r}+i p_{i}\right)\right)\left(s-\left(p_{r}-i p_{i}\right)\right)} \\
\theta & =\left[\begin{array}{llll}
k_{0} & z & p_{r} & p_{i}
\end{array}\right]^{T}
\end{aligned}
$$

with the true parameters being $\theta^{0}=\left[\begin{array}{llll}.2 & .5 & -.25 & .3\end{array}\right]^{T}$ during this run. The sampling noise distribution, $p(\tau)$, is known and given by Eq. (25) with $n=2$ and $a=$ $T / 2 / \sqrt{3} \approx .2887$. The input and measurement noise are the same as in Sec. 5.1. 
Table 1

Results for identification of model (24). Mean value and standard deviation of $\hat{\theta}=[\hat{n} \hat{a}]$ and $\hat{\sigma}=\hat{a} / \sqrt{3(\hat{n}+1)}$ for six sets of true parameters, $\theta^{0}$. In each of the six blocks, the top row shows the true parameter values. The following two rows are the results for BCLS estimation, mean value and standard deviation. The last two rows are the corresponding results for ML estimation. The two lowest blocks have true pdf's outside the model class, which is why no true values of $n$ and $a$ are given.

\begin{tabular}{|c|ccc||ccc|}
\hline & $n$ & $a$ & $\sigma$ & $n$ & $a$ & $\sigma$ \\
\hline \hline$\theta^{o}$ & 0 & 0.5 & $1 / \sqrt{12} \approx 0.2887$ & 0 & $1 / \sqrt{12} \approx 0.2887$ & $1 / 6 \approx 0.1667$ \\
\hline $\mathrm{E}\left[\hat{\theta}^{B C L S}\right]$ & 0.2145 & 0.4683 & 0.2599 & 1.1818 & 0.3759 & 0.1682 \\
$\operatorname{Std}\left[\hat{\theta}^{B C L S}\right]$ & 0.7360 & 0.0678 & 0.0489 & 1.6815 & 0.1186 & 0.0649 \\
\hline $\mathrm{E}\left[\hat{\theta}^{M L}\right]$ & 0.0437 & 0.4801 & 0.2719 & 0.7073 & 0.3660 & 0.1671 \\
$\operatorname{Std}\left[\hat{\theta}^{M L}\right]$ & 0.0789 & 0.0233 & 0.0170 & 0.8184 & 0.0662 & 0.0224 \\
\hline \hline$\theta^{o}$ & 2 & 0.5 & $1 / 6 \approx 0.1667$ & 2 & $1 / \sqrt{12} \approx 0.2887$ & $1 /(6 \sqrt{3}) \approx 0.0962$ \\
\hline $\mathrm{E}\left[\hat{\theta}^{B C L S}\right]$ & 1.1386 & 0.3752 & 0.1676 & 1.6816 & 0.2618 & 0.1018 \\
$\operatorname{Std}\left[\hat{\theta}^{B C L S}\right]$ & 1.6608 & 0.1088 & 0.0592 & 1.9972 & 0.1553 & 0.0607 \\
\hline $\mathrm{E}\left[\hat{\theta}^{M L}\right]$ & 0.6420 & 0.3766 & 0.1732 & 2.4947 & 0.3015 & 0.1011 \\
$\operatorname{Std}\left[\hat{\theta}^{M L}\right]$ & 0.6439 & 0.0753 & 0.0250 & 2.1080 & 0.0866 & 0.0205 \\
\hline \hline$\theta^{o}$ & $\rightarrow \infty$ & - & $1 / 6 \approx 0.1667$ & $\rightarrow \infty$ & - & $1 /(6 \sqrt{3}) \approx 0.0962$ \\
\hline $\mathrm{E}\left[\hat{\theta}^{B C L S}\right]$ & 1.8175 & 0.3267 & 0.1281 & 1.8807 & 0.1958 & 0.0752 \\
$\operatorname{Std}\left[\hat{\theta}^{B C L S}\right]$ & 2.0357 & 0.1607 & 0.0678 & 2.1966 & 0.1425 & 0.0530 \\
\hline $\mathrm{E}\left[\hat{\theta}^{M L}\right]$ & 1.0115 & 0.3589 & 0.1515 & 3.4188 & 0.2696 & 0.0802 \\
$\operatorname{Std}\left[\hat{\theta}^{M L}\right]$ & 1.0415 & 0.0855 & 0.0297 & 2.4361 & 0.0877 & 0.0219 \\
\hline
\end{tabular}

The result for the ML and LS estimation is shown in Table 2, and it is clear that both methods give unbiased estimates with small variances.

Table 2

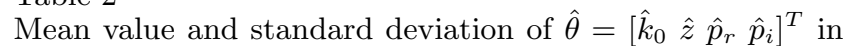
the system (27).

\begin{tabular}{|c|cccc|}
\hline Model $(27)$ & $k_{0}$ & $z$ & $p_{r}$ & $p_{j}$ \\
\hline$\theta^{o}$ & 0.2 & 0.5 & -0.25 & 0.3 \\
\hline $\mathrm{E}\left[\hat{\theta}^{B C L S}\right]$ & 0.2000 & 0.5012 & -0.2504 & 0.3001 \\
$\operatorname{Std}\left[\hat{\theta}^{B C L S}\right]$ & 0.0025 & 0.0135 & 0.0019 & 0.0029 \\
\hline $\mathrm{E}\left[\hat{\theta}^{M L}\right]$ & 0.1992 & 0.4966 & -0.2462 & 0.3001 \\
$\operatorname{Std}\left[\hat{\theta}^{M L}\right]$ & 0.0071 & 0.0319 & 0.0052 & 0.0068 \\
\hline
\end{tabular}

\subsection{Unknown second order OE model with unknown jit- ter pdf}

The previous example is extended by letting the jitter noise pdf be unknown. That is, consider the $\mathcal{M}_{J O E}$ problem in $(2 \mathrm{c})$. The system $g_{\theta}(t)$ is given by $(27)$ and the sampling noise pdf, $p_{\theta}(\tau)$, is given by (25), with $\theta=\left[\begin{array}{llllll}n & a & k_{0} & p_{r} & p_{i} & z\end{array}\right]^{T}$ and the true parameters being

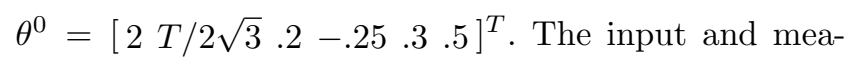
surement noise are the same as in Sec. 5.1.

From the two previous sections, we know that $n$ and $a$ were hard to estimate, whereas $k_{0}, z, p_{r}$ and $p_{j}$ posed no problem. We expect to see similar things for the joint problem. The results are given in Table 3 . The BCLS estimates are good for all parameters, $(\hat{\sigma}=0.11)$. The extremely low variances are mainly due to a course evaluation grid in the $6 \mathrm{D}$ parameter space, see Section 4.5. In this example we also did not use the ML estimate, because of the issues also discussed in Section 4.5.

\section{Conclusions}

The stochastic jitter noise in the model $y_{k}=s(k T+$ $\left.\tau_{k} ; \theta\right)+v_{k}$ should not be neglected and included in the measurement noise $v_{k}$. The bias compensated least squares estimator describes both the effects of jitter and the remedy:

$\hat{\theta}^{B C L S}=\arg \min _{\theta} \sum_{f}\left|Y(f)-\int S(\psi ; \theta) \varphi(-\psi ; \theta) d_{N}(f-\psi) \mathrm{d} \psi\right|^{2}$.

This criteria minimizes the LS distance between measurement TDFT and the model's FT, taking leakage and alias effects into account via a convolution with the normalized Dirichlet kernel $d_{N}(f-\psi)$. The characteristic function $\varphi(-\psi ; \theta)$ of the jitter noise implies a scaling of the signal model $S_{c}(f)$. By neglecting this, a bias in the estimation would result.

A second order compensation of jitter effects leads to the 
Table 3

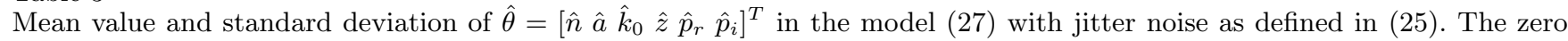
standard deviation depends on the discretization step where the correct grid point was always estimated.

\begin{tabular}{|c|cccccc|}
\hline Model $(25),(27)$ & $n$ & $a$ & $k_{0}$ & $z$ & $p_{r}$ & $p_{j}$ \\
\hline$\theta^{\circ}$ & 2 & $\frac{T}{2 \sqrt{3}} \approx 0.2887$ & 0.2 & 0.5 & -0.25 & 0.3 \\
\hline $\mathrm{E}\left[\hat{\theta}^{B C L S}\right]$ & 2.008 & 0.2938 & 0.2000 & 0.5000 & -0.2500 & 0.3000 \\
$\operatorname{Std}\left[\hat{\theta}^{B C L S}\right]$ & 1.471 & 0.0728 & 0 & 0 & 0 & 0 \\
\hline
\end{tabular}

WLS estimator (20) and the asymptotic maximum likelihood estimator (22). The estimators were illustrated on simulated examples for continuous time OE system identification, with known or with parametrized jitter distribution. The results are very promising and a clear motivation to develop dedicated algorithms for the case of jitter noise. E.g. efficient Gauss-Newton algorithms would be useful, where also unknown noise models (BoxJenkins structures) could be targeted.

\section{References}

[1] Ivar Bilinskis and Arnolds Mikelsons. Randomized Signal Processing. Prentice Hall, 1992.

[2] Frida Eng and Fredrik Gustafsson. System identification using measurements subject to stochastic time jitter. In IFAC World Congress, July 2005.

[3] Frida Eng and Fredrik Gustafsson. Bias compensated least squares estimation of continuous time output error models in the case of stochastic sampling time jitter. In IFAC Symp. on Syst. Ident. (SYSID), March 2006.

[4] Ronald Aylmer Fisher. On the mathematical foundations of theoretical statistics. Philosophical Transactions of the Royal Society of London, Series A., 222:309-368, 1922.

[5] Jonas Gillberg and Fredrik Gustafsson. Frequency-domain continous-time AR modeling using non-uniformly sampled measurements. In IEEE Int. Conf. on Acoust., Speech, Signal Processing, 2005.

[6] Jonas Gillberg and Lennart Ljung. Frequency-domain identification of continous-time ARMA models from sampled data. In IFAC World Congress, July 2005.

[7] Jonas Gillberg and Lennart Ljung. Frequency-domain identification of continuous-time output error models from sampled data. In IFAC World Congress, July 2005.

[8] Thomas Kailath, Ali H Sayed, and Babak Hassibi. Linear Estimation. Prentice Hall, 2000.

[9] Lennart Ljung. System Identification - Theory for the User, 2nd edition. Prentice Hall, 1999.

[10] Farokh Marvasti, editor. Nonuniform Sampling: Theory and Practice. Kluwer Academic Publishers, 2001.

[11] Rik Pintelon and Johan Schoukens. System Identification: A Frequency Domain Approach. IEEE Press, 2001.

[12] Rik Pintelon and Johan Schoukens. ML identification of closed-loop systems in a specified frequency band. In IFAC World Congress, July 2005. 196.

[13] Johan Schoukens, Yves Rolain, and Rik Pintelon. Analysis of windowing/leakage effects in frequency response function measurements. In IFAC World Congress, July 2005.

[14] T Michael Souders, Donald R Flach, Charles Hagwood, and Grace L Yang. The effects of timing jitter in sampling systems. IEEE Trans. Instrum. Meas., February 1990.
[15] Frans Verbeyst, Yves Rolain, Johan Schoukens, and Rik Pintelon. System identification approach applied to jitter estimation. In Instrumentation and Measurement Technology Conference, pages 1752 - 1757, April 2006. 